\title{
Mapping the ultrafast vibrational dynamics of all-trans and 13-Cis retinal isomerization in Anabaena Sensory Rhodopsin
}

\author{
Partha Pratim Roy ${ }^{1 *}$, Rei Youshizumi ${ }^{2}$, Hideki Kandori ${ }^{2}$ and Tiago Buckup ${ }^{1, *}$ \\ ${ }^{1}$ Physikalisch-Chemisches Institut, Ruprecht-Karls Universitat Heidelberg, Germany \\ ${ }^{2}$ Department of Life Science and Applied Chemistry, Nagoya Institute of Technology, Japan
}

\begin{abstract}
The ground and excited state evolution of fingerprint vibrational modes of all-trans-and 13-cis-retinal are mapped by impulsive vibrational spectroscopy. All-trans-retinal shows slower frequency shift dynamics in the excited state in comparison to 13-cis-retinal.
\end{abstract}

\section{Introduction}

Anabaena sensory rhodopsin (ASR) is a microbial retinal protein that comprises a retinal chromophore in two ground state (GS) conformations: all-trans, 15-anti (AT) and 13-cis, 15-syn (13C). The relative content of these isomers can be tuned by external light adaptation [1]. Protein environment and the conformation of the GS isomer are known to be the most crucial factors that determine the reaction dynamics of retinal systems. In this context, ASR offers the distinctive advantage to compare the dynamics of AT and 13C isomers within the same protein environment. Notably, 13C isomer shows almost one order of magnitude faster dynamics [1,2] compared to AT. Although theoretical studies [3] pointed out that the topology of ES potential energy surface is different for AT and 13C, it still lacks direct experimental evidence. In this study, we applied Pump-degenerate four wave mixing (Pump-DFWM) [4] to map the evolution of fingerprint vibrational modes during the isomerization of AT and $13 \mathrm{C}$ isomers. In particular, the pure vibrational signatures of GS and photo-intermediate $\left(\mathrm{K}_{\mathrm{AT}}\right.$ and $\mathrm{K}_{\mathrm{Cis}}$ ) of each isomer were disentangled. Moreover, the excited state evolution of the $\mathrm{C}=\mathrm{C}$ stretching and $\mathrm{CH}_{3}$ rocking mode of $13 \mathrm{C}$ were found to exhibit a faster frequency shift compared to AT isomer.

\section{Experimental details}

The experimental set-up used for Pump-DFWM measurements has been explained in detail previously [4]. In brief, all pulses ( $<15 \mathrm{fs}$ ) were generated using non-collinear optical parametric amplifiers. The actinic pump (AP) spectrum was made resonant to GS absorption of the isomers whereas the DFWM spectrum was resonant to the photo-

\footnotetext{
* Corresponding author: tiago.buckup@pci.uni-heidelberg.de
} 
induced absorption of ASR (Fig. 1(a)). The non-oscillatory contributions were subtracted [5] from each DFWM transient before FFT (Fig. 2(a)-(b)) was performed.

(a)

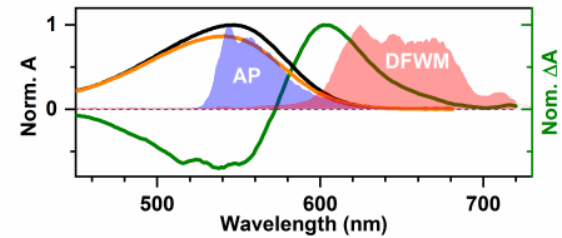

(b)

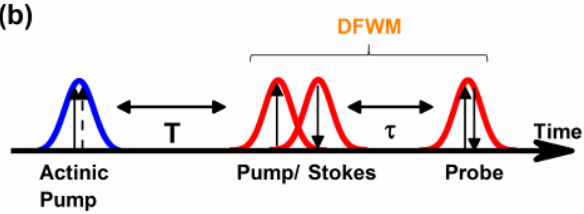

Fig. 1. (a) The spectrum of actinic pump (AP) and DFWM beams are represented by green and blue filled curves respectively. Former is resonant to ground state absorption of AT (black line) and 13C (red line) isomers of ASR and later is resonant to photo-induced absorption (green line). (b) Schematic representation of pulse sequence of Pump-DFWM experiment.

\section{Results and Discussion}

\subsection{Ground state modes}

Pure DFWM experiment, i.e. with AP blocked, delivers the pure GS signal. The DFWM signal, probed at $590 \mathrm{~nm}$, shows the characteristic vibrational frequencies (Fig. 2a) of the GS isomers both in dark adapted (DA) and light adapted (LA) conditions [5] which contain almost pure AT (97\%) and major percentage of 13C isomer (64\%) respectively. The modes around $1000,1100-1300$ and $1500 \mathrm{~cm}^{-1}$ are usually assigned to $\mathrm{CH}_{3}$ rock, $\mathrm{C}-\mathrm{C}$ and $\mathrm{C}=\mathrm{C}$ stretching vibrations. The blue shifts of the $\mathrm{CH}_{3}$ rock and $\mathrm{C}=\mathrm{C}$ stretching modes from DA to LA ASR (Fig. 2a) are in good agreement with previous studies [2]. Also, the C-C stretching modes appear at $1164,1229 \mathrm{~cm}^{-1}$ for DA and $1180 \mathrm{~cm}^{-1}$ for LA ASR are very similar to bacteriorhodopsin (bR) $\left(1165 / 1230 \mathrm{~cm}^{-1}\right.$ and $\left.1190 \mathrm{~cm}^{-1}\right)$. In addition, the modes around $800-1000 \mathrm{~cm}^{-1}$, which are mostly assigned to hydrogen-out-of-plane (HOOP) mode, shows significant difference in FFT amplitude in DA and LA conditions. In particular, the mode around $805 \mathrm{~cm}^{-1}$, which was assigned to $\mathrm{C}_{14}-\mathrm{H}$ wag for $\mathrm{bR}$, shows stronger amplitude in LA ASR compared to DA ASR (Fig. 2a). This observation is consistent with nonresonant spontaneous Raman spectra (not shown). Raman activity of HOOP mode indicates distorted non-planar geometry. Thus, it hints to larger distortion of retinal chain near the active double bond $\left(\mathrm{C}_{13}=\mathrm{C}_{14}\right)$ of $13 \mathrm{C}$ compared to AT although no direct evidence of different extent of distortion for AT and 13C isomer is known for ASR till now. However, this larger pre-strain in GS of 13C isomer compared to AT can explain the accelerated isomerization dynamics similar to 11-cis isomer of visual Rhodopsin. In order to extract the vibrational signature of sub-ns K-photo-intermediate, Pump-DFWM measurement at an AP delay $\mathrm{T}=100 \mathrm{ps}$ were carried out. A constrained multi-Gaussian fit gives the pure vibrational signatures of $\mathrm{GS}$ as well as $\mathrm{K}$-photo-products $\left(\mathrm{K}_{\mathrm{AT}}\right.$ and $\left.\mathrm{K}_{13 \mathrm{C}}\right)$.

\subsection{Excited state evolution}

To map the ES frequency evolution of the vibrational modes, the AP delay was varied from $\mathrm{T}=-40$ fs to $1 \mathrm{ps}$. Subtraction of GS contribution by constrained fitting at each $\mathrm{T}$ delay allowed us to derive the pure $\mathrm{ES}$ evolution of $\mathrm{C}=\mathrm{C}$ stretch and $\mathrm{CH}_{3}$ rock mode for AT isomer (Fig. 2c-e). Both modes show a rapid blue shift $\left(\sim 20 \mathrm{~cm}^{-1}\right)$ within $200 \mathrm{fs}$ and then a slower red shift to form $\mathrm{K}_{\mathrm{AT}}$. A similar trend was observed for LA ASR (Fig. 2d-f). The ES 

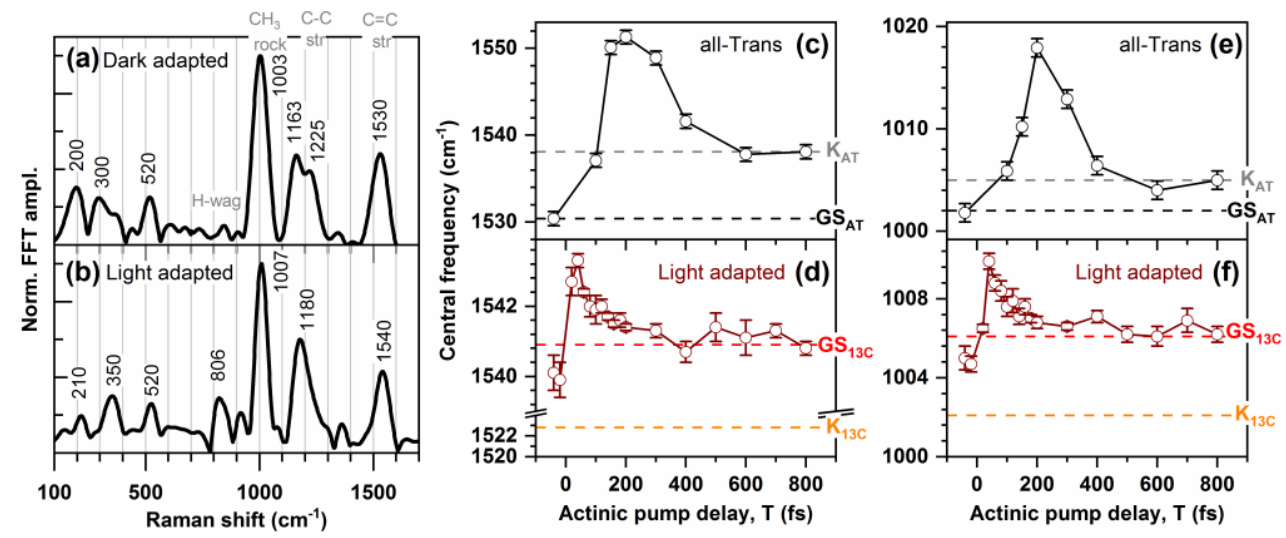

Fig. 2. The pure GS vibrational spectra of $\mathrm{AT}$ (a) and $13 \mathrm{C}$ (b) isomer. The excited state evolution of $\mathrm{C}=\mathrm{C}$ stretching $(\mathrm{c}, \mathrm{d})$ and $\mathrm{CH}_{3}$ rock modes $(\mathrm{e}, \mathrm{f})$ obtained for the pure $\mathrm{AT}$ isomer $(\mathrm{c}, \mathrm{e})$ and $\mathrm{LA}(\mathrm{d}, \mathrm{f})$ form ASR. Centre frequencies of pure GS (AT and 13C), LA GS and pure K-photo intermediates $\left(\mathrm{K}_{\mathrm{AT}}\right.$ and $\left.\mathrm{K}_{13 \mathrm{C}}\right)$ are shown by black, red, brown, grey and orange dotted lines respectively in each graph.

evolution of both $\mathrm{C}=\mathrm{C}$ stretching and $\mathrm{CH}_{3}$ rock modes of LA also shows a blue shift followed by a red shift (Fig. 2d-f), but within a much faster time scale in comparison to AT (Fig. 2c-e). Here, it is important to note that the major signal contributor to the pumpDFWM signal of LA ASR is the 13C isomer, but also it contains minor contributions of both isomers in the GS as well as of the AT in the ES, which makes the extraction of the exact $13 \mathrm{C}$ evolution more challenging. Nevertheless, the faster frequency shifts of LA ASR is interpreted here to be originated mainly by the $13 \mathrm{C}$ isomer, the major component. The different time scales in the frequency shifts for the AT and $13 \mathrm{C}$ isomers correlate to the ES lifetimes, which are reported as 750 and $110 \mathrm{fs}$, respectively [2].

The blue-shift of $\mathrm{C}=\mathrm{C}$ stretching and $\mathrm{CH}_{3}$ rock modes has been interpreted as the reduction of the effective $\pi$-conjugation length during the formation of the twisted transition state via the rotation around the $\mathrm{C}_{13}=\mathrm{C}_{14}$ bond. Therefore, the slower blue shift of AT hints at a delayed formation of the twisted TS and it corroborates previous QM/MM calculations [3] which predicts a barrier in the excited state trajectory of AT isomer.

\section{References}

1. A. Cheminal, J. Leonard, S. Y. Kim, K.H. Jung, H. Kandori, and S. Haacke Phys. Chem. Chem. Phys. 17, 25429 (2015).

2. A. Wand, R. Rozin, T. Eliash, K. H. Jung, M. Sheves, S. Ruhman, J. Am. Chem. Soc. 133, 20922 (2011).

3. A. Strambi, B. Durbeeja, N. Ferré, M. Olivucci, PNAS 129, 8644 (2010).

4. T. Buckup and M. Motzkus, Annu. Rev. Phys. Chem. 65, 39 (2015).

5. D. Agathangelou, Y. Gonzalez, M. C. Marín, P. P. Roy, J. Brazard, H. Kandori, K. Jung, J. Léonard, T. Buckup, N. Ferré, M. Olivucci, S. Haacke, Faraday Discussion, 207, 55 (2018). 